BULL. AUSTRAL. MATH. SOC.

VOL. I (1969), 403-416.

\title{
On certain abelian-by-nilpotent varieties
}

\section{J. M. Brady, R. A. Bryce and John Cossey}

\begin{abstract}
We show that, whenever $m, n$ are coprime, each subvariety of the

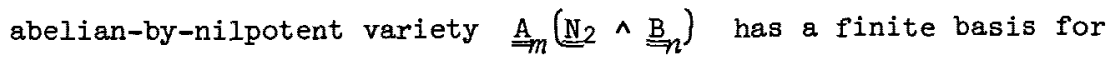
its laws. We further show that the just non-Cross subvarieties of $\stackrel{\mathrm{A}}{\Rightarrow}\left(\underline{\mathbb{N}}_{2} \wedge \stackrel{\mathrm{B}}{=}\right)$ are precisely those already known.
\end{abstract}

\section{Introduction}

In his papers [2, 3] Graham Higman has described techniques for answering a range of questions regarding certain varieties of groups. Here we work out the details which enable us to prove the following two results.

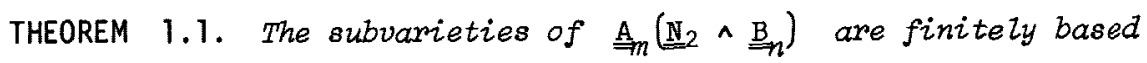
whenever $m, n$ are coprime.

(As usual $\stackrel{\mathrm{A}}{\rightarrow}_{n}, \underline{\mathrm{N}}_{2}, \underline{\mathrm{B}}_{n}$ are respectively the varieties of: abelian groups of exponent dividing $m$, groups of class at most 2 and groups of exponent dividing $n$. We shall follow the terminology of Hanna Neumann [5]).

THEOREM 1.2. Let $\stackrel{\mathrm{T}}{=}$ be the variety generated by the non-abelian group of order $q^{3}$ and exponent $q$ when $q$ is an odd prime, and by the dihedral grow of order 8 when $q$ is 2 . Then, whenever $m, n$ are coprime, the just non-Cross subvarieties of $\mathrm{A}_{m}\left(\underline{\mathrm{N}}_{2} \wedge \underline{\mathrm{B}}_{n}\right)$ are precisely $\stackrel{\mathrm{A}}{\Rightarrow} \stackrel{\mathrm{T}}{q}(p|m, q| n)$.

Recall that a just non-Cross variety is one not generated by a finite group every proper subvariety of which is generated by a finite group. By now Theorem 1.1 requires little motivation; and we refer the reader to

Received 28 July 1969. 
Kovács and Newman [4] for the motivation behind Theorem 1.2. In [3] Higman asks another question relevant to the above varieties: if $\underline{\underline{V}}$ is a subvariety of $\underline{A}_{m}\left(\underline{N}_{2} \wedge \underline{B}_{n}\right)$ where $m, n$ are coprime, and $f(n)=\left|F_{n}(\underline{V})\right|$, is $f: z^{+} \rightarrow z^{+}$so defined CREAM ? We believe we can give an affirmative answer to this question; as the proof is long and tedious we shall, however, not reproduce it here.

Since $\underline{\mathrm{A}}_{m}\left(\underline{\underline{N}}_{2} \wedge \underline{\mathrm{B}}_{n}\right)$ has a finite basis for its laws [2, Theorem 3.1], Theorem 1.1 is proved if the subvarieties of $\stackrel{\mathrm{A}}{m}_{m}\left(\underline{\mathrm{N}}_{2} \wedge \mathrm{B}_{n}\right)$ satisfy the descending chain condition, and to show this it is enough to prove

LEMMA 1.3. If $\left\{G_{i}: i \in Z^{+}\right\}$is an infinite sequence of critical groups in $\mathrm{A}_{m}\left(\underline{\underline{N}}_{2} \wedge \underline{\mathrm{B}}_{n}\right)$, with $m, n$ coprime, then for some $i$, $G_{i} \in \operatorname{var}\left\{G_{i+1}, G_{i+2}, \ldots\right\}$.

The description of the critical groups of $\stackrel{A}{\Rightarrow}\left(\underline{N}_{2} \wedge \stackrel{B}{B_{n}}\right)$ given in $\S 2.7$ of [3] will be taken as read. There Higman uses the description to show that to prove CREAM for $\underline{A} \underline{\underline{B}}$, where $\underline{\underline{B}}$ is locally finite of exponent prime to $m$, it suffices to show it for $\underline{A} B$ for each prime divisor $p$ of $m$. The same argument shows that it is enough to prove Theorems 1.1 and 1.2 for the case when $m$ is prime: henceforth we assume $m$ is a prime $p$.

To each non-nilpotent critical group in $\underline{\underline{A}}(\underline{\underline{N}} 2 \wedge \underline{\underline{B}})$, Higman associates a unique irreducible linear group over $G F(p)$, the field of $p$ elements. The proof of the theorems now depends on a complete classification of such irreducible linear groups; it is well known that they have cyclic centre, so the classification is given by the following two lemmas whose proofs are given in $\$ 2$ (the second is a corollary of 2.4 ).

LEMMA 1.4. If $\left\{K_{i}: i \in Z^{+}\right\}$is an infinite sequence of groups of class 2 and exponent dividing $n$ with cyclic centres, then for some $i, j$ with $i$ less than $j, K_{i}$ is isomorphic to a central factor of $K_{j}$ (i.e. $K_{i}$ is isomorphic to a subgroup of $K_{j}$ which together with its centralizer in $K_{j}$, this subgroup generates $K_{j}$ ). 
LEMMA 1.5. If $K$ is a group of class 2 with cyclic centre and of order prime to $p$, then all faithful irreducible representations of $K$ over $G F(p)$ are linearly isomorphic.

Proof of Lemma 1.3. Let $K_{i}$ be the irreducible linear group associated with $G_{i}$ for each $i$ in $z^{+}$. By Lemma 1.4 there exist $i, j$ with $i$ less than $j$ such that, as abstract group, $K_{i}$ is a subgroup of $K_{j}$ with the centre $z\left(K_{i}\right)$ contained in $z\left(K_{j}\right)$. Let $M$ be the space over $G F(p)$ on which $K_{j}$ acts, and consider an irreducible component $M_{0}$ of $M_{K_{i}}$. Since each element of $z\left(K_{j}\right)$ acts fixed-point-free on $M, M_{0}$ is faithful. Consequently even as linear group $K_{i}$ is contained in $K_{j}$, by Lemma 1.5 , and thus $G_{i}$ is a subgroup of $G_{j}$. Lemma 1.3 , and with it Theorem 1.1, is therefore proved.

\section{Proofs of Lemmas 1.4 and $\mathbf{1 . 5}$}

We begin by determining the structure of groups of class 2 with cyclic centre. Since a nilpotent group is a direct product of groups of prime power order, it suffices to consider $q$-groups for some prime $q$.

THEOREM 2.1. (cf. M.F. Newman [6]). A finite class 2 q-group with cyclic centre is a central product either of two-generator subgroups with cyclic centre or of two-generator subgroups with cyclic centre and a cyclic subgroup.

Proof. Suppose that $K$ satisfies the hypotheses. The proof is by induction on the minimal number of generators for $K$. If $K$ requires either one or two generators, there is nothing to prove. Hence suppose that $K$ cannot be generated by two elements and that the result is true for such groups with fewer generators: note that $K$ is then not abelian. Choose an arbitrary set of generators for $k$, say $k=x, y, u, \ldots$. Since $K^{\prime}$, the derived group of $K$, is a cyclic $q$-group and $K^{\prime}$ is generated by all the commutators $[x, y],[x, u], \ldots$ it follows that $K^{\prime}$ is generated by one of them, say by $[x, y]$. We will show that $K$ is generated by $\left\{x, y, u^{\prime}, \ldots\right\}$ where $u^{\prime}, \ldots$ all centralize $x$ and $y$ : for there is an integer $r$ such that 


$$
[x, u]=[x, y]^{r}=\left[x, y^{r}\right]
$$

whence $\left[x, u y^{-r}\right]=1$, and an integer $s$ such that

$$
\left[y, \operatorname{lay}^{-x}\right]=[y, x]^{8}=\left[y, x^{8}\right]
$$

whence $\left[y, u y^{-r} x^{-8}\right]=1$. Then $u^{\prime}=u y^{-r} x^{-8}$ commutes with $x, y$ and $\langle x, y, u\rangle=\left\langle x, y, u^{\prime}\right\rangle$. Similarly one replaces the other elements of the given generating set.

In this way we have $K$ expressed as a central product of $\langle x, y\rangle$ and a subgroup $K_{1}$ on fewer generators; $\langle x, y\rangle$ and $K_{1}$ both have cyclic centres, and so induction concludes the proof. Note that the amalgamations of the centres of the central factors must be as large as possible.

Our need now is to determine all two-generator groups of class 2 with cyclic centre.

THEOREM 2.2. The q-groups of class 2 on two generators with cyclic centre comprise the following list:

$$
\begin{aligned}
& Q(\alpha, \beta)(2 \beta \leq \alpha):\left\langle a, b: a^{q^{\alpha}}=b^{q^{\beta}}=1, a^{q^{\alpha-\beta}}=[a, b]\right\rangle ; \\
& Q(\alpha, \beta)(\beta \leq \alpha<2 \beta):\left\langle a, b: a^{q^{\alpha}}=b^{q^{\beta}}=1, a^{q^{\beta}}=[a, b]^{q^{2 \beta-\alpha}},\right. \\
& \text { and if } q=2 \text { we have as well } \quad[a, b, a]=[a, b, b]=1) ;
\end{aligned}
$$

$$
\begin{aligned}
R(\beta) \quad(1 \leq \beta) \quad:\langle a, b & : a^{2^{\beta+1}}=b^{2^{\beta+1}}=1, a^{2^{\beta}}=[a, b]^{2^{\beta-1}} \\
& \left.=b^{2^{\beta}},[a, b, a]=[a, b, b]=1\right\rangle .
\end{aligned}
$$

Proof. Let $X$ be a two generator group of class 2 with cyclic centre. Choose a generating pair $\{a, b\}$ for $X$ where the order of $a$ is $q^{\alpha}$, that of $b, q^{\delta}(\alpha \geq \delta)$ and that of $[a, b], q^{\beta}$.

If $a^{q^{\lambda}} \in Z(X)$ then $1=\left[a^{q^{\lambda}}, b\right]=[a, b]^{q^{\lambda}}$ whence $\lambda \geq \beta$. Treat $b$ similarly, so that

$$
Z(X)=\left\langle a^{q^{\beta}}, b^{q^{\beta}},[a, b]\right\rangle
$$

Since $Z(X)$ is cyclic, two cases arise. 
Case 1. $z(X)=\left\langle a^{q^{B}}\right\rangle$. There exists an integer $r$ such that

$$
[a, b]=a^{r q}{ }^{\beta}
$$

whence $[a, b]^{q^{\lambda}}=a^{r q^{\beta+\lambda}} ;$ consequently $1=a^{r q^{2 \beta}}$ and $q^{\alpha} \| r q^{2 \beta}$ so that $\alpha \geq 2 \beta$ and $q^{\alpha-2 \beta} \| r$. Write $r=q^{\alpha-2 \beta} s(q / 8)$ and choose $t$ so that st $\equiv 1\left(\bmod q^{\alpha}\right) ;$ then

$$
\left[a, b^{t}\right]=[a, b]^{t}=a^{q^{\alpha-\beta}}
$$

Since $\left\{a, b^{t}\right\}$ is a generating set for $X$ we may as well assume that $a, b$ as given satisfy

$$
[a, b]=a^{q^{\alpha-\beta}}
$$

Now there is an integer $u$ such that $b^{q^{\beta}}=a^{u q^{\beta}}$ so that, for all integers $v$,

$$
\left(b a^{v}\right)^{q^{\beta}}=b^{q^{\beta}} a^{v q^{\beta}}[a, b]^{v\left(q_{2}^{q^{\beta}}\right)}=a^{u q^{\beta}+v\left(q^{\beta}+\left(\begin{array}{c}
q^{\beta} \\
2
\end{array}\right) q^{\alpha-\beta}\right)} .
$$

We aim to choose $v$ so that $\left(b a^{v}\right)^{q^{\beta}}=1$, and this can certainly be done if the congruence

$$
u q^{\beta}+v\left(q^{\beta}+q^{\alpha-\beta}\left(\begin{array}{l}
q^{\beta} \\
2
\end{array}\right)\right) \equiv 0\left(\bmod q^{\alpha}\right)
$$

can be solved for $v$. In case $q$ is odd this can always be done; and when $q=2$ it can be done in all cases except $\alpha=2, \beta=1, u \neq 0$ (mod 2) (this exception corresponding to the quaternion group $Q_{8}$ ). Notice that $a, b a^{\nu}$ satisfy the relation (*). Hence $X$ is a homomorphic image of $Q(\alpha, \beta)$ and since $Q(\alpha, \beta)$ has cyclic centre and its derived group has the same order as that of $X$, the kernel of the homomorphism is 1 .

Case 2. $Z(X)=([a, b])$. By copying the earlier argument one deduces that $\alpha \leq 2 \beta$ and that $b$ may be changed so that 


$$
[a, b]^{q^{2 \beta-\alpha}}=a^{q^{\beta}}
$$

Again one looks for $v$ so that $\left(b a^{v}\right)^{q^{\beta}}=1$, and if $[a, b]^{u}=b^{q^{\beta}}, v$ must satisfy

$$
u+v\left(q^{2 \beta-\alpha}+\left(\begin{array}{c}
q^{\beta} \\
2
\end{array}\right)\right) \equiv 0 \quad\left(\bmod q^{\beta}\right)
$$

When $q$ is odd such a $v$ always exists; and if $q=2$ one may be found in all cases except $\alpha=\beta+1$. Hence one gets either $Q(\alpha, \beta)$ or $R(\beta)$.

COROLLARY 2.3. Define $Q(\beta)=Q(\beta, \beta)$ and let $c=\left\langle c: c^{q^{\alpha}}=1\right\rangle$ $(\alpha \geq \beta)$. Form the central product $D(\alpha, \beta)$ of $Q(\beta)$ and $C$ with the amalgamation

$$
c^{q^{\alpha-\beta}}=[a, b]
$$

Then $Q(\alpha, \beta) \cong\langle a c, b\rangle$. Also when $q=2$ and $\alpha=\beta+1 \quad R(\beta) \cong\langle b a, b c\rangle$ in $D(\beta+1, \beta)$ and this is the unique copy of $R(\beta)$ in $D(\beta+1, \beta)$.

Proof. This first statement requires showing that $a c, b$ satisfy the appropriate relations, and that $\left|\langle a c, b\rangle^{\prime}\right|=q^{\beta}$; we omit the details.

For the second case write $\left(\begin{array}{l}2^{\beta} \\ 2\end{array}\right)=2^{\beta-1} u$, so that $u$ is odd, and choose $v$ so that $u v \equiv 1\left(\bmod 2^{\alpha}\right)$. Put $a_{1}=b a^{v}, b_{1}=b^{u} c: a_{1}, b_{1}$ each have order $2^{\alpha}$, and

$$
a_{1}^{2^{\beta}}=[a, b]^{v\left(\begin{array}{c}
2^{\beta} \\
2
\end{array}\right)}=[a, b]^{2^{\beta-1}}=c^{2^{\beta}}=b_{1}^{2^{\beta}} .
$$

Hence $\left\langle a_{1}, b_{1}\right\rangle \cong R(\beta)$.

Finally note that if $D_{0}$ is a subgroup of $D=D(\beta+1, \beta)$ isomorphic to $R(\beta)$, then $D_{0}$ has index 2 in $D$. Now $\left|D_{0}: \Phi\left(D_{0}\right)\right|=4$, $|D: \Phi(D)|=8$ (where $\Phi$ as usual denotes the Frattini subgroup); and since $\Phi(D) \leq D_{0}, \Phi\left(D_{0}\right) \leq \Phi(D)$ whence $\Phi(D)=\Phi\left(D_{0}\right)$. It follows that there are 7 a priori choices for $D_{0}$, and noting that the canonic generators $a^{\prime}, b^{\prime}$ for $D_{0}$ have order $2^{\alpha}$ and $a^{\prime} b^{\prime}$ does also, one 
discards 6 of these. (Since $u, v$ are odd and $a^{2}, b^{2}, c^{2} \in \Phi\left(D_{0}\right)$, $\left(a_{1}, b_{1}\right)=(a, b)$

REMARK. If $K$, a group of class 2 with cyclic centre, is written as a central product of $Q(\alpha, \beta)$ 's, $R(\beta)$ 's and perhaps one cycle, it is, up to isomorphism, uniquely determined by the number of times each central factor in this decomposition is repeated. For, each automorphism of $Z(Q)$, where $Q$ is a $Q(\alpha, \beta)$ or an $R(\beta)$ or cyclic, is the restriction of an automorphism of $Q$; and hence no matter how the amalgamations are performed one gets the same group.

The crucial result of this section is:

THEOREM 2.4. Let $K$ be a group of class 2 with cyclic centre and $E$ a field whose characteristic does not divide the order of $K$. Then the faithful irreducible representations of $K$ over $E$ are linearly isomorphic.

The proof of this fact requires a number of steps: we first show that it is sufficient to establish the result for algebraically closed fields, and then that only the groups of Theorem 2.2 really matter.

THEOREM 2.5. Let $X$ be a finite group, $E$ a field and $T_{1}, T_{2}$ faithful irreducible representations of $X$ over $E, T_{1}, T_{2}$ are linearly isomorphic if and only if some composition factor of $T_{1}{ }^{*}$ is linearly isomorphic to a composition factor of $T_{2}{ }^{*}$ (where $E^{*}$ denotes the algebraic closure of E).

Proof. The 'only if' part is trivial, so suppose $T_{1} E^{*}$ has a composition factor $T_{11}$ linearly isomorphic to a composition factor $T_{21}$ of $T_{2}{ }^{E^{*}}$. Since $X$ is finite, $T_{11}$ and $T_{21}$ may be written in some finite extension field $F$ of $E$. Choose $F$ so that $|F: E|>\operatorname{dim} T_{11}$; the first part of the proof of (29.7) in Curtis and Reiner [1] can then be routinely amended to show that $T_{11}$ and $T_{21}$ are linearly isomorphic F-representations. That is $T_{1}{ }^{F}, T_{2}{ }^{F}$ have a linearly isomorphic composition factor.

Now $F$ may be chosen as a splitting field for $X$ and also as a finite, normal extension of $E$, so that (70.15) of [1] tells us that $T_{1}{ }^{F}, T_{2}{ }^{F}$ are completely reducible, say 


$$
T_{1}^{F}=T_{11} \oplus \ldots \oplus T_{1 m}, \quad T_{2}^{F}=T_{21} \oplus \ldots \oplus T_{2 n},
$$

and that $T_{1 i}, T_{2 i}$ are all faithful representations of $X$. Now there exists $U \in G L\left(\operatorname{dim} T_{11}, F\right)$ such that

$$
T_{11}(X)^{U}=T_{21}(X) \text {. }
$$

Define the automorphism $\alpha$ of $X$ by

$$
T_{21}(x \alpha)=T_{11}(x)^{U}, x \in X,
$$

and the representation $T_{3}$ of $X$ over $F$ by

$$
T_{3}(x)=T_{1}\left(x \alpha^{-1}\right), \quad x \in X .
$$

Clearly $T_{3}$ is faithful and irreducible. It is easy to calculate that $T_{2}{ }^{F}, T_{3}{ }^{F}$ have the composition factor $T_{21}$ in common. Consequently $T_{2}, T_{3}$ are equivalent by (29.6) of [1] (being irreducible). But $T_{3}(X)=T_{1}(X)$ so that $T_{1}, T_{2}$ are linearly isomorphic as required.

In order to reduce the problem to the case when $K$ is one of the groups of Theorem 2.2 we need the following manifestation of a well known result (adapt the proof of $[1,(51.3)]$ ).

THEOREM 2.6. Let $X, Y$ be finite groups and $H=X \times Y ; E$ an arbitrary field and $M$ an EH-module. Let $M_{1}$ be an absolutely irreducible submodule of $M_{X}$. Then $M$ is irreducible if and only if $M \cong M_{1} \# M_{2}$ for any irreducible submodule $M_{2}$ of $M_{Y}$.

COROLLARY 2.7. If $X, Y$ are groups each of which has just one linear isomorphism class of faithful irreducible representations over an algebraically closed field $E$, then every central product of $X$ and $Y$ with cyclic centre has the some property. (Note that this includes the case $X \times Y, X, Y$ of coprime orders.)

Proof. If $H_{0}$ is a central product of $X$ and $y$ then there is an epimorphism $H=X \times Y \rightarrow H_{0}$. Let $M, N$ be faithful irreducible modules over $E$ for $H_{0} ; M, N$ therefore can be regarded as irreducibles of $H$. Consequently, by the theorem, $M=M_{1} \# M_{2}, N=N_{1} \# N_{2}$ where $M_{1}, N_{1}$ are faithful irreducibles of $X$ and $M_{2}, N_{2}$ faithful irreducibles of $Y$. 
Now if $T_{i}, U_{i}$ are the representations for $X, Y$ afforded by $M_{i}, N_{i}(i=1,2)$ respectively, then there exist non-singular linear transformations $V, W$ such that

$$
T_{1}(X)^{V}=T_{2}(X), U_{1}(Y)^{W}=U_{2}(Y) .
$$

Put $V_{0}=V \otimes W$ and one easily checks that

$$
\left(T_{1}(X) U_{1}(Y)\right)^{V_{0}}=T_{2}(X) U_{2}(Y)
$$

as required.

Theorem 2.5 and Corollary 2.7 have therefore reduced the proof of Theorem 2.4 to the case when $K$ is either $Q(\alpha, \beta), R(\beta)$ or cyclic, and $E$ is algebraically closed; the result is well known for cyclic groups and we treat the remainder in a number of steps starting with $K=Q(B)(=Q(B, B))$.

LEMMA 2.8. Al2 faithful irreducibles of the groups Q(B) over a field whose characteristic is not $q$ are linearly isomorphic.

Proof. As we have observed we assume the ground field $E$ to be closed. Construct representations $T_{i}$ of the subgroup $A=\langle a,[a, b]\rangle$ of $Q(B)$ as follows: the kernel of each $T_{i}$ is $\langle a\rangle$, and

$$
T_{i}([b, a])=\xi^{i}, \quad i \in\left\{1, \ldots, q^{\beta-1}\right\}, q \backslash i
$$

where $\xi$ is a primitive $q^{\beta}$ th root of 1 . For each $i$ and each $x \in Q(\beta)-A$, the representations $T_{i}$ and $T_{i}^{(x)}: h \rightarrow T_{i}\left(x^{-1} h x\right)$ of $A$ are distinct, therefore inequivalent, and hence each induced representation $T_{i}^{Q(B)}$ is irreducible $\left(\left[1,(45.2)^{\prime}\right]\right)$. Moreover if $i \neq j$, the representations $T_{i}$ and $T_{j}(x)$ are distinct, therefore inequivalent, for each $x \in Q(\beta)$, and so $T_{i}^{Q(B)}$ and $T_{j}^{Q(B)}$ are inequivalent $([1,(45.6)])$. The number of these $T_{i}^{Q(\beta)}$ 's is $\left(q^{\beta}-1\right)-\left(q^{\beta-1}-1\right)=q^{\beta-1}(q-1)$. We show now that this accounts for all faithful irreducibles of $Q(B)$ over $E$.

LEMMA 2.9. Let $X$ be a q-group of class 2 with cyclic centre of 
order $q^{\beta}$. The nomber of faithful absolutely irreducible representations of $X$ is $q^{\beta-1}(q-1)$.

Proof. We may assume $X$ non-abelian as otherwise the result is well known. Notice that the unique minimal normal subgroup (the monolith) $O X$ of $X$ is the socle of $X^{\prime}$. If elements $x, y$ of $X$ are conjugate modulo $\sigma X$, then there exists $t \in X$ and $z \in \sigma X$ such that

$$
x z=y^{t}
$$

Now there exists $u \in X$ such that $z=[x, u]$ whence $x=y^{t u^{-1}}$, and $x, y$ are conjugate. Moreover a central element cannot be conjugate modulo $\sigma X$ to a non-central element. It follows that if $N$ is the number of non-central conjugacy classes of $X$, the number of conjugacy classes in $X / \sigma X$ is $N+|Z(X) / \sigma X|=N+q^{\beta-1}$. Hence the number of faithful absolutely irreducible representations of $X$ is $\left(N+q^{\beta}\right)-\left(N+q^{\beta-1}\right)=q^{\beta-1}(q-1)$ as required.

Continuing with the proof of Lemma 2.8 we find that a basis for the modules underlying the $T_{i} Q(\beta)$ may be constructed as follows: if $\left\{z_{i}\right\}$ is a basis for the $A$-module $T_{i},\left\{z_{i} \otimes b^{j}: 0 \leq j \leq q^{\beta}-1\right\}$ is a basis for $T_{i}^{Q(B)}$. The matrices representing $a, b$ are then easily determined:

$$
\begin{aligned}
& T_{i}{ }^{Q(\beta)}: b \rightarrow\left(\begin{array}{lll}
{ }^{1} & & \\
& \ddots & \\
1 & &
\end{array}\right), \\
& T_{i}^{Q(B)}: a \rightarrow\left(\begin{array}{llll}
1 & & & \\
& \xi^{i} & & \\
& \xi^{2 i} & \\
& & \ddots & \xi^{\left(q^{\beta}-1\right) i}
\end{array}\right) .
\end{aligned}
$$

It is immediately clear that the $T_{i}^{Q(B)}$ are faithful and linearly isomorphic.

We now use Corollary 2.3 to complete the proof of Theorem 2.4. Notice that $Q(\alpha, \beta)$ is a subgroup of $D(\alpha, \beta)$ in such a way that the monoliths 
coincide. The regular representation of $D(\alpha, \beta)$ restricted to $Q(\alpha, \beta)$ contains a copy of the regular of $Q(\alpha, \beta)$. Consequently every faithful irreducible of $Q(\alpha, \beta)$ arises by restricting a faithful irreducible of $D(\alpha, \beta)$; moreover it follows from Theorem 2.6 that if $M$ is a faithful irreducible $D(\alpha, \beta)$-module then $M_{Q(\beta)}$ is irreducible and, since all subspaces of $M$ admit $C, M_{Q(\alpha, \beta)}$ is irreducible. It is therefore easy to write down the matrices representing the irreducibles of $Q(\alpha, \beta)$ and see that they form a single linear group.

The case of $R(\beta)$ is similar: note that by Corollary 2.7 all faithful irreducibles of $D(\beta+1, \beta)$ are linearly isomorphic; and since there is precisely one copy of $R(\beta)$ in $D(\beta+1, \beta)$, by Corollary 2.3, all faithful irreducibles of $R(\beta)$ are linearly isomorphic. The proof of Theorem 2.4 is therefore complete.

It remains to prove Lemma 1.4 .

Proof of Lemma 1.4. The two-generator groups with cyclic centre in $\underline{N}_{2} \wedge \underline{B}_{n}$ are finite in number; call them $L_{1}, \ldots, L_{N}$ say. By Theorem 2.1 each $K_{i}$ is a central product of $L_{j}{ }^{\prime} s$, possibly not uniquely; nevertheless with a fixed decomposition of $K_{i}$ as such a central product containing $r_{i j}$ copies of $L_{j}$ associate the $N$-tuple $\left(r_{i l}, \ldots, r_{i N}\right)$. It is well known that the set of all $N$-tuples with ordering by components is partially well ordered. Consequently for some $i<j, r_{i l} \leq r_{j l}$ $(1 \leq Z \leq N)$. Thus $K_{i}$ is a central factor of $K_{j}$, proving Lemma 1.4 (see the Remark following the proof of Corollary 2.3).

\section{The proof of Theorem 1.2}

Let $\underline{\mathrm{V}} \leq \mathrm{A}_{\mathrm{A}}\left(\underline{\underline{N}}_{2} \wedge \underline{\mathrm{B}}_{n}\right)$ be Just non-Cross and let $\underline{\underline{\mathrm{X}}}$ be the closed class of linear groups associated with $\underline{\underline{v}}$ à Za Higman [2, 54$]: \underline{\underline{U}}(\underline{\underline{x}})=\underline{\underline{v}}$. Now $\underline{\underline{X}}$ is infinite and every proper closed subclass is finite; and each group in $\underline{\underline{X}}$ is a direct product of groups described in Theorem 2.1. There are but finitely many cyclic groups and two generator groups with cyclic centre in $\underline{\underline{N}}_{2} \wedge \underline{B}_{n}$ and consequently arbitrarily large central powers of one of them, say $Q$, occur in $\underline{\underline{X}}$. Since each group in $\underline{\underline{X}}$ has at most one cyclic 
central factor, $Q$ is not cyclic.

Write

$$
\underline{x}_{0}=\left\{C_{n}: n \in z^{+}\right\}
$$

where $C_{n}$ is a central product with cyclic centre of $n$ copies $Q_{1}, \ldots, Q_{n}$ of $Q$; then $\underline{\underline{X}}$ is the closure of $\underline{x}_{0}$.

LEMMA 3.1. For each $n>1, C_{n}$ contains, as linear factor, the central product of $n-1$ copies of $Q(1)$.

Proof. We show first that $C_{n}$ contains, as linear factor, the central product with cyclic centre of groups $S_{1}, \ldots, S_{n}$ each isomorphic to a $Q(\alpha, 1)$ or to $R(1)$. For, if $\left\{a_{i}, b_{i}\right\}$ is a canonic generating set for $Q_{i}$ consider $H_{i}=\left\langle a_{i}, b_{i}^{q^{\beta-1}}\right\rangle$ and set $k_{n}=H_{1} H_{2} \ldots H_{n}$. One easily checks that the centre $z\left(H_{i}\right)$ of $H_{i}$ is $\left\langle a_{i}^{q},\left[a_{i}, b_{i}\right]^{q^{\beta-1}}\right\rangle$ and that

$$
z\left(K_{n}\right)=z\left(H_{1}\right) \ldots z\left(H_{n}\right), K_{n}^{\prime}=\left\langle\left[a_{i}, b_{i}\right]^{q^{\beta-1}}\right\rangle \quad(1 \leq i \leq n) .
$$

Notice also that $z\left(K_{n}\right)$ is the Frattini subgroup $\Phi\left(K_{n}\right)$ of $K_{n}$.

Now let $M$ be a faithful irreducible module for $C_{n}$ over $G F(p)$ and consider an irreducible component $M_{0}$ of $M_{K_{n}}$. Since the kernel of $M_{0}$ must avoid $K_{n}^{\prime}$ it is easy to see that

$$
\operatorname{ker} M_{0} \leq z\left(K_{n}\right)=\Phi\left(K_{n}\right) \text {. }
$$

$M_{0}$ is a faithful and irreducible module for $P_{n}=K_{n} / \operatorname{ker} M_{0} \cdot P_{n}$ has cyclic centre, derived group exponent $q$, and it requires $2 n$ generators since $\Phi\left(P_{n}\right)=\Phi\left(K_{n}\right) / \operatorname{ker} M_{0}$. Hence $P_{n}$ is a central product as asserted in the first sentence of the proof.

Suppose now that $S_{1}, \ldots, S_{n}$ have as canonic generating sets $\left\{a_{i}^{\prime}, b_{i}^{\prime}\right\}$; we write $\alpha_{i}$ for the order of $a_{i}^{\prime}$ and suppose 
$\alpha_{1} \geq \alpha_{i}(i \geq 1)$. If $\alpha_{i}>1 \quad(i>1$ we have that the subgroup $\left(a_{1}, q^{\alpha_{1}-\alpha_{i}}, a_{i}^{\prime}, b_{i}^{\prime}\right\rangle$ has cyclic centre $\left\langle a_{1} q^{\alpha_{1}-\alpha_{i}}\right\rangle$ and is a central product of $\left(a_{1}{ }^{q^{\alpha_{1}-\alpha_{i}}}\right)$ and $\left\langle a_{i}^{\prime}, b_{i}^{\prime}\right\rangle$. Corollary 2.3 then yields that $\left\langle a_{1} q^{\alpha_{1}-\alpha_{i}}, a_{i}^{\prime}, b_{i}^{\prime}\right\rangle$ is a central product of $\left\langle a_{1}, q^{\alpha_{1}-\alpha_{i}}\right\rangle$ and a subgroup $J_{i}$ isomorphic to $Q(1)$. Clearly $I_{n}=J_{2} \ldots J_{n}$ (where $J_{i}=S_{i}$ if $\left.\alpha_{i}=1\right)$ is a central product with cyclic centre of $n-1$ copies of $Q(1)$. Since $Z\left(I_{n}\right) \leq Z\left(P_{n}\right), I_{n}$ is a component of $P_{n}$, and the lemma is proved. Finally note that Higman [2, Ex. 4.10] has shown that if $Q \cong Q(1)$, $\underline{y}_{0}=\left\{C_{n}: n \in Z^{+}\right\}$, where $C_{n}$ is a central product with cyclic centre of $n$ copies of $Q$ and $\underline{\underline{Y}}$ is the closure of $\underline{\underline{Y}}_{0}$, then $\underline{\underline{U}}(\underline{\underline{Y}})=\underline{\underline{A}} \underline{\underline{\underline{T}}} q$ and that this is just non-Cross. Hence, under the assumptions on $\underline{\underline{X}}, \underline{\underline{U}}(\underline{\underline{X}})=\frac{A}{A_{p}} \underline{\underline{T}} q$ as required.

\section{References}

[1] Charles W. Curtis and Irving Reiner, Representation theory of finite groups and associative algebras (Interscience, New York, 1962).

[2] Graham Higman, "Some remarks on varieties of groups", Quart. J. Math., Oxford (2) 10 (1959), 165-178.

[3] Graham Higman, "The orders of relatively free groups", Proc. Internat. Conf. Theory of Groups, Austral. Nat. Univ., Canberra, 1965 (Gordon and Breach, New York, 1967).

[4] L.G. Kovács and M.F. Newman, "Just non-Cross varieties", Proc. Internat. Conf. Theory of Groups, Austral. Nat. Univ., Canberra, 1965 (Gordon and Breach, New York, 1967).

[5] Hanna Neumann, Varieties of groups (Ergebnisse der Mathematik, und ihrer Grenzgebiete, Band 37, Springer-Verlag, Berlin, Heidelberg, New York, 1967). 
[6] M.F. Newman, "On a class of nilpotent groups", Proc. London Math. Soc. (3) $10(1960), 365-375$.

Australian National University, Canberra, ACT. 